\title{
Structural Parameter Identification in Time Domain using Extended Kalman Filter Method with Weighted Global Iteration
}

\author{
Xiang-Jian WANG ${ }^{1, a^{*}}$ and Jie CUI ${ }^{2,3, b}$ \\ ${ }^{1}$ Key Laboratory of Earthquake Engineering and Engineering Vibration, Institute of Engineering \\ Mechanics, China Earthquake Administration, Harbin 150080, China \\ ${ }^{2}$ Department of Civil Engineering, Guangzhou University, Guangzhou 510006, China \\ ${ }^{3}$ Earthquake Engineering Research and Test Centre, Guangzhou University, Guangzhou 510405, \\ China \\ a wang.xiangjian@163.com, b jcui2009@hotmail.com
}

Supported by: the National Science Foundation of China under Grant No. 51208478

\begin{abstract}
Keywords: structural parameter identification; extended Kalman filter method; memory fading filter technique; weighted global iteration; white noise

Abstract: A structural parameter identification method is proposed to identify the stiffness parameters and damping coefficient and to estimate the unmeasured responses in time domain. The unique feature of this technique is that it requires only the displacement responses at all or most of dynamic degree-offreedoms (DDOFs). This new method is a combination of the extended Kalman filter technique with weighted global iteration (EKF-WGI) technique, memory fading filter (MFF) technique and Runge-Kutta (R-K) method, and it is called as MFEKF-WGI by the authors. The parameter identification and the response estimation are carried out using the proposed method by a numerical example. Three noise contaminated cases of the displacement responses are considered. The robustness, efficiency and accuracy of the new technique are verified by the results. However, the proposed method requires more sample points and smaller time interval for the accuracy of estimation, and requires a complete response time histories for the estimation of unmeasured responses.
\end{abstract}

\section{Introduction}

Dynamic behavior of complicated system often needs to be investigated by system identification (SI) to meet some requirements. SI methods in structural dynamics, in general solve inverse problems to identify properties of a structure from measured data. With the rapid progress in the field of computing technique, use of efficient mathematical tools and experimental technique, SI has become important topic in the field of civil engineering, because SI techniques play an important role in investigating and reducing gaps between the building and their design models. This is also true in structural health monitoring (SHM) for damage detection. And the SI methods are generally used to obtain the dynamic behavior of the structures, to know current status of structural systems against dynamic loads such as earthquake etc.

For dynamic system of civil engineering, the mathematical model is usually known. The SI process is only for the structural parameters, it is called parameter identification. A great amount of researches with various methodologies have been conducted in parameter identification, such as artificial neural network approach (ANN) [1], wavelet analysis method [2], Fourier transform based method [3], finite element-based Iterative Least-squares methods (ILS) [4,5,6,7], adaptive forgetting through multiple models (AFMM) coupled with auto-regression with exogenous variables (ARX) [8], frequency domain decomposition (FDD) [9], natural excitation technique coupled with eigen-system realization algorithm (NExT-ERA) [10], Random decrement technique (RDT) [11], extended Kalman filter technique (EKF) [12,13], and so on.

Since structural physical parameters for damage estimation is intuitive and identified in time domain without cut-off error [6], structural physical parameter identification in time domain become a major branch of SI. Many researches $[14,15]$ show that the extended Kalman filter technique with weighted 
global iteration (EKF-WGI) is a good method for structural physical parameter identification in time domain. EKF-WGI is suitable for not only linear parametric system, but also nonlinear parametric system, and doesn't need to measure the dynamic response of every dynamic degree of freedom (DDOF). In this paper, the memory fading filter technique (MFF) and Runge-Kutta method (R-K) are introduced, an improved method, MFEKF-WGI, is proposed for physical parameter identification in time domain.

\section{Extended Kalman Filter Method with Weighted Global Iteration (EKF-WGI)}

For physical parameter identification of dynamic system, the physical parameters are extended into the state vector, and estimated using Kalman filter method, that is called extended Kalman filter (EKF). The EKF technique is explained as follows [16,17]:

The governing equation of motion of a multiple degree of freedom (MDOF) system can be written in matrix form as

$$
\mathbf{M}+\mathbf{C x}+\mathbf{K x}=\mathbf{f}(t)
$$

where $\mathbf{K}, \mathbf{C}$ and $\mathbf{M}=$ stiffness, damping and mass matrix of the structure, respectively; $\mathbf{x}, \mathbf{\alpha}$ and $=$ displacement, velocity and acceleration vectors, respectively; $\mathbf{f}(t)=$ excitation force vector.

The EKF method is an iterative process to estimate the state of the structural system based on the measured input and output data. A state equation is needed for the EKF technique.

Defining the state vector as

$$
\begin{aligned}
& \mathbf{X}(t)=\left[\begin{array}{llll}
\mathbf{X}_{1}(t) & \mathbf{X}_{2}(t) & \mathbf{X}_{3}(t) & \mathbf{X}_{4}(t)
\end{array}\right]^{\mathrm{T}} \\
& \mathbf{X}_{1}(t)=\left[\begin{array}{c}
x_{1}(t) \\
x_{2}(t) \\
\mathrm{M} \\
x_{n}(t)
\end{array}\right], \mathbf{X}_{2}(t)=\left[\begin{array}{c}
\alpha(t) \\
\alpha_{2}(t) \\
\mathrm{M} \\
\boldsymbol{\alpha}_{n}(t)
\end{array}\right], \mathbf{X}_{3}=\left[\begin{array}{c}
k_{1} \\
k_{2} \\
\mathrm{M} \\
k_{l}
\end{array}\right], \mathbf{X}_{4}=\left[\begin{array}{c}
c_{1} \\
c_{2} \\
\mathrm{M} \\
c_{l}
\end{array}\right]
\end{aligned}
$$

where $\mathbf{X}(t)=$ state vector at time $t ; \mathbf{X}_{1}(t)$ and $\mathbf{X}_{2}(t)=$ displacement and velocity vectors at time $t$, respectively; $\mathbf{X}_{3}(t)$ and $\mathbf{X}_{4}(t)=$ stiffness and damping vectors in element-level, respectively. $n=$ total number of DDOFs; $l=$ total number of elements; $x_{i}(t)$ and $\left.t\right)=$ displacement and velocity at the $i$ th DDOF at time $t ; k_{i}$ and $c_{i}=$ stiffness and damping parameters in element-level that need to be identified, and they are assumed not to change with time during the identification process. And the mass of every DDOF is assumed as known and not to change with time. Eq. 1 can be expressed in a state equation as

$$
\mathbf{X}=\left\{\begin{array}{l}
\mathbf{X}_{1} \\
\mathbf{x}_{2} \\
\mathbf{x}_{3} \\
\mathbf{x}_{4}
\end{array}\right\}=\left\{\begin{array}{c}
\mathbf{X}_{2} \\
\mathbf{M}^{-1}\left[\mathbf{f}(t)-\mathbf{C}\left(\mathbf{X}_{4}\right) \cdot \mathbf{X}_{2}-\mathbf{K}\left(\mathbf{X}_{3}\right) \cdot \mathbf{X}_{1}\right] \\
\mathbf{0} \\
\mathbf{0}
\end{array}\right\}
$$

where $\mathbf{K}\left(\mathbf{X}_{3}\right)$ denotes stiffness matrix is the function of the state vector $\mathbf{X}_{3} ; \mathbf{C}\left(\mathbf{X}_{4}\right)$ denotes damping matrix is the function of the state vector $\mathbf{X}_{4}$. In general, Eq. 4 can be written as

$$
\$(t)=\mathbf{g}[\mathbf{X}(t), \mathbf{f}(t), t]+\mathbf{L}(t) \cdot \mathbf{w}(t)
$$


Eq. 5 is the nonlinear continuous state equation, where $\mathbf{X}(t)=$ continuous state vector, $\mathbf{g}[\bullet]$ denotes the nonlinear function of $\mathbf{X}(t), \mathbf{L}(t)=$ matrix of system noise, $\mathbf{w}(t)=$ system noise vector.

Suppose the dynamic response of the structure is measured at time $t_{k+1}=(k+1) \Delta t$, where $\Delta t=$ time interval between measurements. Then the observation equation at time $t_{k+1}=(k+1) \Delta t$ can be expressed as

$$
\mathbf{Z}_{k+1}=\mathbf{h}\left[\mathbf{X}_{k+1}, k+1\right]+\mathbf{V}_{k+1}
$$

Eq. 6 is the nonlinear discrete observation equation, where $\mathbf{X}_{k+1}=\mathbf{X}\left(t_{k+1}\right)=$ discrete state vector, $\mathbf{h}[\bullet]$ denotes the nonlinear function of $\mathbf{X}_{k+1}, \mathbf{V}_{k+1}=$ observation noise.

Some assumptions are given as follows:

$$
\begin{aligned}
& \left.\left.E[\mathbf{w}(t)]=0, E\left[\mathbf{V}_{k}\right]=0, \operatorname{Cov}[\mathbf{w}(t), \mathbf{w}(\tau)]=\mathbf{Q}_{\mathbf{w}}(t), \operatorname{Cov} \mid \mathbf{V}_{k}, \mathbf{V}_{j}\right]=\mathbf{R}_{k} \boldsymbol{\delta}_{k j}, \operatorname{Cov} \mid \mathbf{w}(t), \mathbf{V}_{j}\right]=\mathbf{0} \\
& E\left[\mathbf{X}_{0}\right]=\overline{\mathbf{X}}_{0}, \operatorname{Var}\left[\mathbf{X}_{0}\right]=E\left[\left(\mathbf{X}_{0}-\overline{\mathbf{X}}_{0}\right)\left(\mathbf{X}_{0}-\overline{\mathbf{X}}_{0}\right)^{T}\right]=\mathbf{P}_{0} \\
& E\left[\mathbf{X}_{0}, \mathbf{w}(t)\right]=0
\end{aligned}
$$

where $\mathbf{Q}_{\mathbf{w}}(t)$ and $\mathbf{R}_{k}=$ covariance matrixes of system noise and observation noise, respectively; $\boldsymbol{\delta}_{k j}=$ Kronecker delta function; $\mathbf{X}_{0}=$ initial value of state vector $\mathbf{X} ; \mathbf{P}_{0}=$ variance matrix of initial value vector $\mathbf{X}_{0}$.

From Eq. 5 and Eq. 6, a iterative process of the EKF technique can be performed in the following steps, starting with the filtered state $\hat{\mathbf{X}}_{k \mid k}$ and its error covariance matrix $\mathbf{P}_{k \mid k}$ from time $t_{k}=k \Delta t$ :

(1) Evaluating the predicted state vector and its error covariance matrix by

$$
\begin{aligned}
& \hat{\mathbf{X}}_{k+1 \mid k}=\hat{\mathbf{X}}_{k \mid k}+\int_{t_{k}}^{t_{k+1}} \mathbf{g}[\mathbf{X}(t), \overline{\mathbf{f}}(k), t] d t \\
& \overline{\mathbf{f}}(k)=[\mathbf{f}(k)+\mathbf{f}(k+1)] / 2 \\
& \mathbf{P}_{k+1 \mid k}=\boldsymbol{\Phi}_{k} \mathbf{P}_{k \mid k} \boldsymbol{\Phi}_{k}^{\mathrm{T}}+\boldsymbol{\Gamma}_{k} \mathbf{Q}_{w, k} \boldsymbol{\Gamma}_{k}^{\mathrm{T}} / \Delta t
\end{aligned}
$$

where $\boldsymbol{\Phi}_{k}$ and $\boldsymbol{\Gamma}_{k}=$ state transition matrix and discrete noise distribution matrix of the system, respectively; for small $\Delta t$, stiffness matrix, damping matrix and input vector are assumed not to change with time, and the following approximately expression can be obtained:

$$
\begin{aligned}
& \boldsymbol{\Phi}_{k}=e^{\mathbf{A}(k) \Delta t} \approx I+\Delta t \cdot \mathbf{A}(k)=I+\Delta t \cdot[\partial \mathbf{g} / \partial \mathbf{X}]_{\mathbf{X}=\hat{\mathbf{X}}_{k \mid k}} \\
& \boldsymbol{\Gamma}_{k}=\boldsymbol{\Phi}_{k} \cdot \mathbf{L}_{k} \cdot \Delta t
\end{aligned}
$$

(2) Calculating the Kalman gain matrix by

$$
\mathbf{K}_{k+1}=\mathbf{P}_{k+1 \mid k} \mathbf{H}_{k+1}^{\mathrm{T}}\left[\mathbf{H}_{k+1} \mathbf{P}_{k+1 \mid k} \mathbf{H}_{k+1}^{\mathrm{T}}+\mathbf{R}_{k+1}\right]^{-1}
$$

$$
\mathbf{H}_{k}=[\partial \mathbf{h} / \partial \mathbf{X}]_{\mathbf{X}=\hat{\mathbf{x}}_{k+1 \mid k}}
$$

where $\mathbf{H}_{k}=$ observation matrix of the system. 
(3) Estimating the filtered state vector and its error covariance matrix by

$$
\begin{aligned}
& \left.\hat{\mathbf{X}}_{k+1 \mid k+1}=\hat{\mathbf{X}}_{k+1 \mid k}+\mathbf{K}_{k+1}\left\{\mathbf{Z}_{k+1}-\mathbf{h} \mid \hat{\mathbf{X}}_{k+1 \mid k}, k+1\right]\right\} \\
& \mathbf{P}_{k+1 \mid k+1}=\left[\mathbf{I}-\mathbf{K}_{k+1} \mathbf{H}_{k+1}\right] \mathbf{P}_{k+1 \mid k}\left[\mathbf{I}-\mathbf{K}_{k+1} \mathbf{H}_{k+1}\right]^{\mathrm{T}}+\mathbf{K}_{k+1} \mathbf{R}_{k+1} \mathbf{K}_{k+1}^{\mathrm{T}}
\end{aligned}
$$

(4) Take the increment $k=k+1$, and return to (1) until $k=m$, where $m$ represents the discrete time $t_{m}$ for the last datum.

The above-mentioned iteration procedure, from $k=0$ to $m$, is called as a local iteration. To obtain the convergent and stable solutions, a weighted global iterative procedure with an objective function can be coupled with the local EKF procedure after the local iteration process is finished.

In the EKF-WGI procedure [16,17], the global iterations are carried out by weighting the error covariance matrix at each global iteration. To start the first global iterations, the initial values of $\hat{\boldsymbol{X}}^{(1)}(0 \mid 0)$ and $\mathbf{P}^{(1)}(0 \mid 0)$ need to be assumed, where superscript (1) represents the first global iteration. Then, using the local EKF procedure, the estimations of $\hat{\mathbf{X}}^{(1)}(m \mid m)$ and $\mathbf{P}^{(1)}(m \mid m)$ are obtained. In the second global iteration, the initial values for $\hat{\mathbf{X}}^{(2)}(0 \mid 0)$ and $\mathbf{P}^{(2)}(0 \mid 0)$ can be set to be:

$$
\hat{\mathbf{X}}^{(2)}(0 \mid 0)=\left[\begin{array}{c}
\hat{\mathbf{X}}_{1}^{(1)}(0 \mid 0) \\
\hat{\mathbf{X}}_{2}^{(1)}(0 \mid 0) \\
\hat{\mathbf{X}}_{3}^{(1)}(m \mid m) \\
\hat{\mathbf{X}}_{4}^{(1)}(m \mid m)
\end{array}\right]=\left[\begin{array}{c}
\hat{\mathbf{X}}^{(1)}(0 \mid 0) \\
\hat{\mathbf{X}}^{(1)}(0 \mid 0) \\
\hat{\mathbf{K}}^{(1)}(m \mid m) \\
\hat{\mathbf{C}}^{(1)}(m \mid m)
\end{array}\right], \mathbf{P}^{(2)}(0 \mid 0)=\left[\begin{array}{cc}
\mathbf{I} & \mathbf{0} \\
\mathbf{0} & w \mathbf{P}_{k c}^{(1)}(m \mid m)
\end{array}\right]
$$

where $w=$ weight used to accelerate the local EKF iteration; $\mathbf{P}_{k c}^{(1)}(m \mid m)=$ error covariance matrix corresponding to the parameters $\hat{\mathbf{K}}$ and $\hat{\mathbf{C}}$ at time $t_{m}$ in the last iteration. The global iterations can be repeated until convergence of the estimated state is obtained.

\section{Memory Fading Filter Technique (MFF)}

In theory, with the increase of the measured data, the filtered state will be estimated accurately, and its error covariance matrix will gradually tends to zero or steady value. But in the practical application, the filtered error is far beyond the allowed range and even tends to infinity. This phenomenon is called filtering divergence [17].

The EKF is an infinite memory filter. To avoid filtering divergence, the MFF technique is introduced to increase the weight of the new measured data and decrease the weight of the old data. The bad influence of the old data will be reduced gradually. From Eq. 12, the MFF technique [17] is expressed as

$$
\mathbf{P}_{k+1 \mid k}=\boldsymbol{\Phi}_{k} \mathbf{P}_{k \mid k} \boldsymbol{\Phi}_{k}^{\mathrm{T}} S+\boldsymbol{\Gamma}_{k} \mathbf{Q}_{w, k} \boldsymbol{\Gamma}_{k}^{\mathrm{T}} / \Delta t
$$

where $S=$ weight, $1.0 \leq S \leq 1.05$. In this paper, the EKF-WGI procedure is coupled with MFF technique, an improved method, MFEKF-WGI, is proposed.

\section{Runge-Kutta Method (R-K)}

For the dynamic system, if the dimension of the state vector, $m$, is no less than $2 n$ ( $n$ is the total number of DDOFs), the vibration problem of MDOF system can be transferred to the initial value 
problem of first order differential equations. The most common and effective solving algorithm is Runge-Kutta method or its improved methods.

The state equation of EKF, Eq. 5, can be written as the initial value form

$$
\left\{\begin{array}{l}
\mathbf{X}(t)=\mathbf{g}[\mathbf{X}(t), \mathbf{f}(t), t]+\mathbf{L}(t) \cdot \mathbf{w}(t) \\
\mathbf{X}\left(t_{0}\right)=\mathbf{X}(0 \mid 0)
\end{array}\right.
$$

In the time interval $\left(t_{k}, t_{k+1}\right)$, the solving formula of Eq. 21 are expressed in discrete form by fourth order R-K method as

$$
\begin{aligned}
& \mathbf{X}(k+1 \mid k)=\mathbf{X}(k \mid k)+\frac{\Delta t}{6}\left(\mathbf{K}_{1}+2 \mathbf{K}_{2}+2 \mathbf{K}_{3}+\mathbf{K}_{4}\right) \\
& \mathbf{K}_{1}=\mathbf{g}\left[\mathbf{X}(k+1 \mid k), \mathbf{f}(k), t_{k}\right] \\
& \mathbf{K}_{2}=\mathbf{g}\left[\mathbf{X}(k+1 \mid k)+\frac{\Delta t}{2} \mathbf{K}_{1}, \overline{\mathbf{f}}(k), t_{k}+\frac{\Delta t}{2}\right] \\
& \mathbf{K}_{3}=\mathbf{g}\left[\mathbf{X}(k+1 \mid k)+\frac{\Delta t}{2} \mathbf{K}_{2}, \overline{\mathbf{f}}(k), t_{k}+\frac{\Delta t}{2}\right] \\
& \mathbf{K}_{4}=\mathbf{g}\left[\mathbf{X}(k+1 \mid k)+\Delta t \mathbf{K}_{3}, \mathbf{f}(k+1), t_{k}+\Delta t\right]
\end{aligned}
$$

In this paper, the predicted state is obtained by fourth order R-K method (Eq. 22).

\section{Numerical Example}

To verify the MFEKF-WGI procedure, a six-story shear-type building, in Fig. 1, is considered. The actual parameter values of the 6-story building are shown in Table 1. And the damping coefficient for stiffness proportional damping, $\beta=0.020914$, is calculated using the modal analysis.

The support excitation is El Centro seismic ground motion, in Fig. 2, and considered to be known. Only the displacement responses are assumed to be measured at each floor. The theoretical structural displacement responses are simulated as the measured responses using the finite element method (FEM). The measured time interval is $0.002 \mathrm{sec}$, the total number of the discrete measured time points is 15590 .

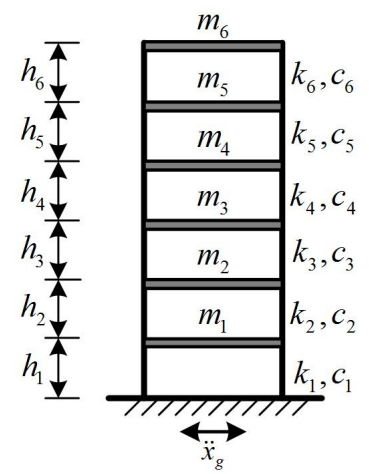

Fig. 1 Six-story shear-type structural model
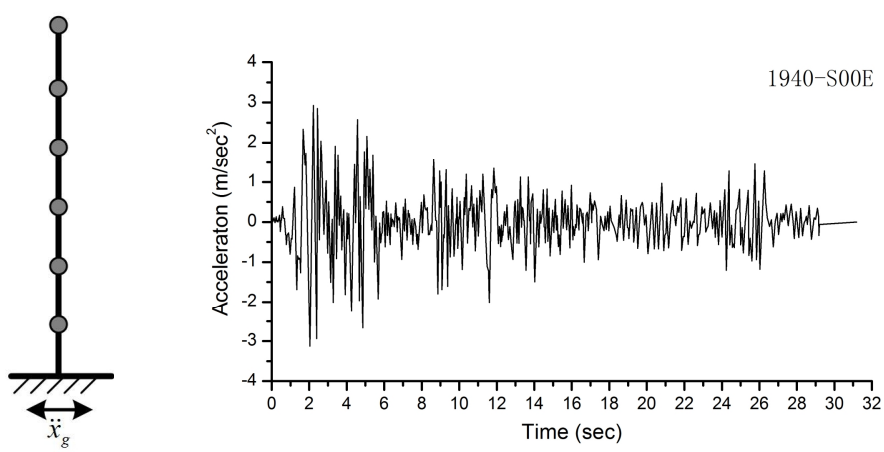

Fig. 2 Time history curve of El Centro ground motion

The task is to identify the structural stiffness parameters, damping coefficient and to estimate the unmeasured responses of all DDOFs using MFEKF- WGI procedure with only the displacement responses. To investigate the effect of measurement noise on the results of state estimation, three levels of white noise are introduced into the theoretical displacement responses. The 5\% noise-contaminated responses are shown in Fig. 3. The noise level is defined as the ratio of noise's RMS (root mean square) 
to theoretical response's RMS, i.e. noise-to-signal ratio. The three considered noise levels are $0 \%, 1 \%$ and $5 \%$ in noise-to-signal ratio, respectively.

Table 1 Actual parameter values of the 6-story building

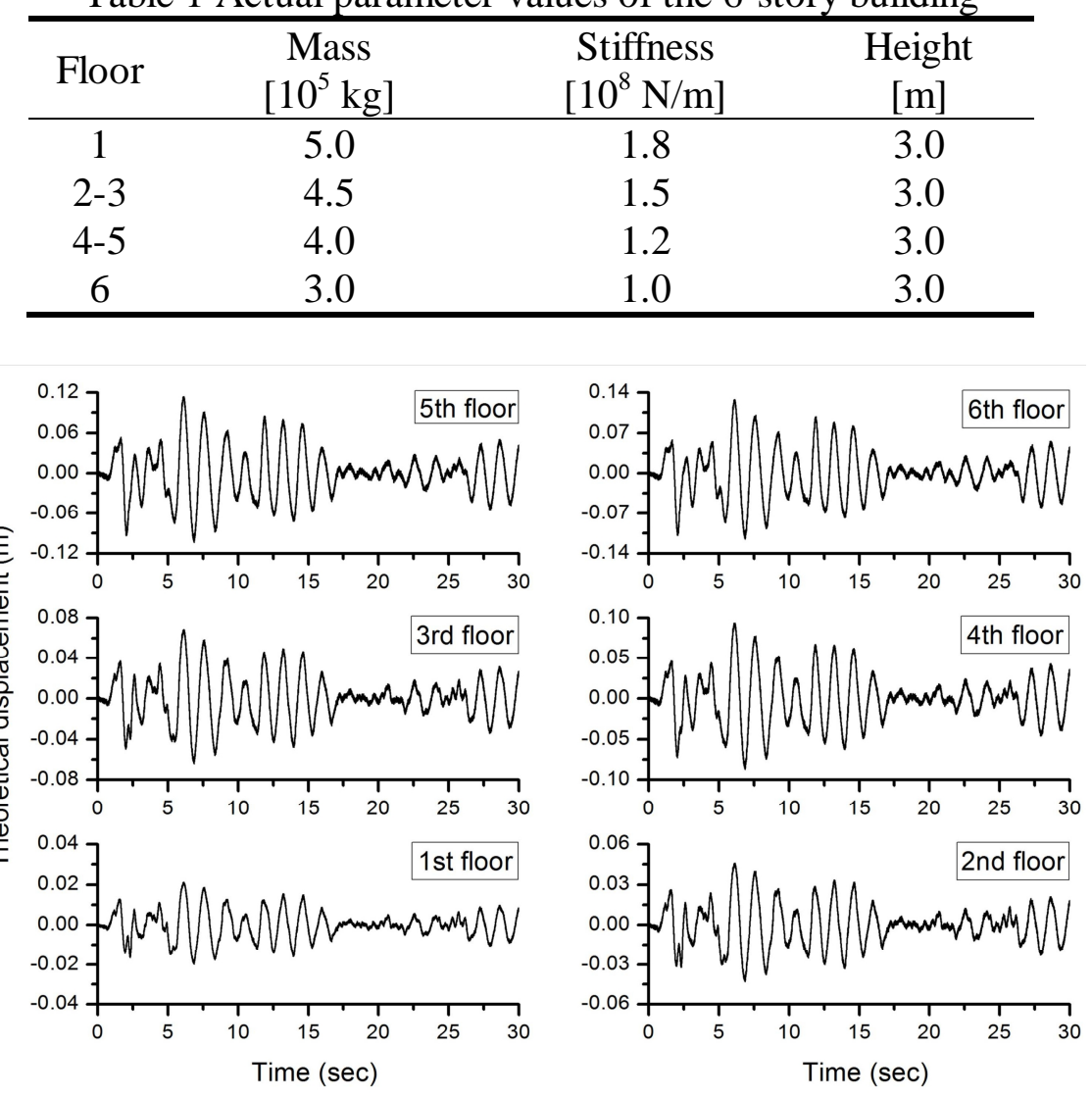

Fig. 3 Time history curves of theoretical displacement with 5\% noise-included case

The state vector $(\mathbf{X})$ and the nonzero elements of the observation matrix $\left(\mathbf{H}_{6 \times 19}\right)$ are

$$
\begin{aligned}
& \mathbf{X}=\left[\begin{array}{c}
\mathbf{X}_{1} \\
\mathbf{M} \\
\mathbf{X}_{4}
\end{array}\right], \mathbf{X}_{1}=\left[\begin{array}{c}
x_{1} \\
\mathbf{M} \\
x_{6}
\end{array}\right], \mathbf{X}_{2}=\left[\begin{array}{c}
\mathbf{x} \\
\mathbf{M} \\
\hat{6}
\end{array}\right], \mathbf{X}_{3}=\left[\begin{array}{c}
k_{1} \\
\mathbf{M} \\
k_{6}
\end{array}\right], \mathbf{X}_{4}=\beta \\
& H_{1,1}=H_{2,2}=H_{3,3}=H_{4,4}=H_{5,5}=H_{6,6}=1.0
\end{aligned}
$$

The total number of global iteration is 2 . The measured responses between $0.002-6.0 \mathrm{sec}$ are used in the first global iteration for the first identification of the parameters, giving $m=3000$. All the measured data are used in the second global iteration for the estimation of the unmeasured responses and the improved identification of the parameters, giving $m=15590$. So the total number of iteration steps is 18590 . The covariance matrix of system noise is $\mathbf{Q}=\mathbf{0}$; The covariance matrix of observation noise is $\mathbf{R}=10^{-2} \times \mathbf{I}$; The weight of global iteration is $w=1000$; And the weight of MFF is $S=1.005$. The initial values of the parameters are $k_{1-5}=1.0 \times 10^{8}, k_{6}=0.8 \times 10^{8}, \beta=0.0160$. The initial values of the state components in the velocity and displacement are zero. The initial values of the filtered error covariance matrix are

$$
\mathbf{P}(0 \mid 0)=\left[\begin{array}{cc}
\mathbf{I}_{12} & \mathbf{0} \\
\mathbf{0} & w \mathbf{I}_{7}
\end{array}\right]
$$


The identified values of the structural physical parameters are listed in Table 2. And the estimated velocity responses compared with the theoretical responses are shown in Fig. 4 (with 5\% noisecontaminated case). Fig. 5 represents the convergence process of parameters identification (with $5 \%$ noise-contaminated case).

Table 2 The identified results of the structural physical parameters

\begin{tabular}{ccccccccc}
\hline & \multirow{2}{*}{ Floor } & \multirow{2}{*}{ Exact } & \multicolumn{2}{c}{$0 \%$ noise } & \multicolumn{2}{c}{$1 \%$ noise } & \multicolumn{2}{c}{$5 \%$ noise } \\
\cline { 3 - 8 } & & & Results & Err. [\%] & Results & Err. [\%] & Results & Err. [\%] \\
\hline & 1 & 1.8 & 1.8000 & 0.00 & 1.7996 & -0.02 & 1.7978 & -0.12 \\
$k_{i}$ & 1.5 & 1.5000 & 0.00 & 1.4996 & -0.03 & 1.4981 & -0.13 \\
{$\left[10^{8} \mathrm{~N} / \mathrm{m}\right]$} & 3 & 1.5 & 1.5000 & 0.00 & 1.4995 & -0.03 & 1.4977 & -0.15 \\
& 4 & 1.2 & 1.2000 & 0.00 & 1.1996 & -0.03 & 1.1980 & -0.17 \\
& 5 & 1.2 & 1.2000 & 0.00 & 1.1997 & -0.02 & 1.1984 & -0.13 \\
$\beta$ & 6 & 1.0 & 1.0000 & 0.00 & 0.9999 & -0.01 & 0.9995 & -0.05 \\
& - & 0.020914 & 0.020914 & 0.00 & 0.020961 & 0.22 & 0.021149 & 1.12 \\
\hline
\end{tabular}

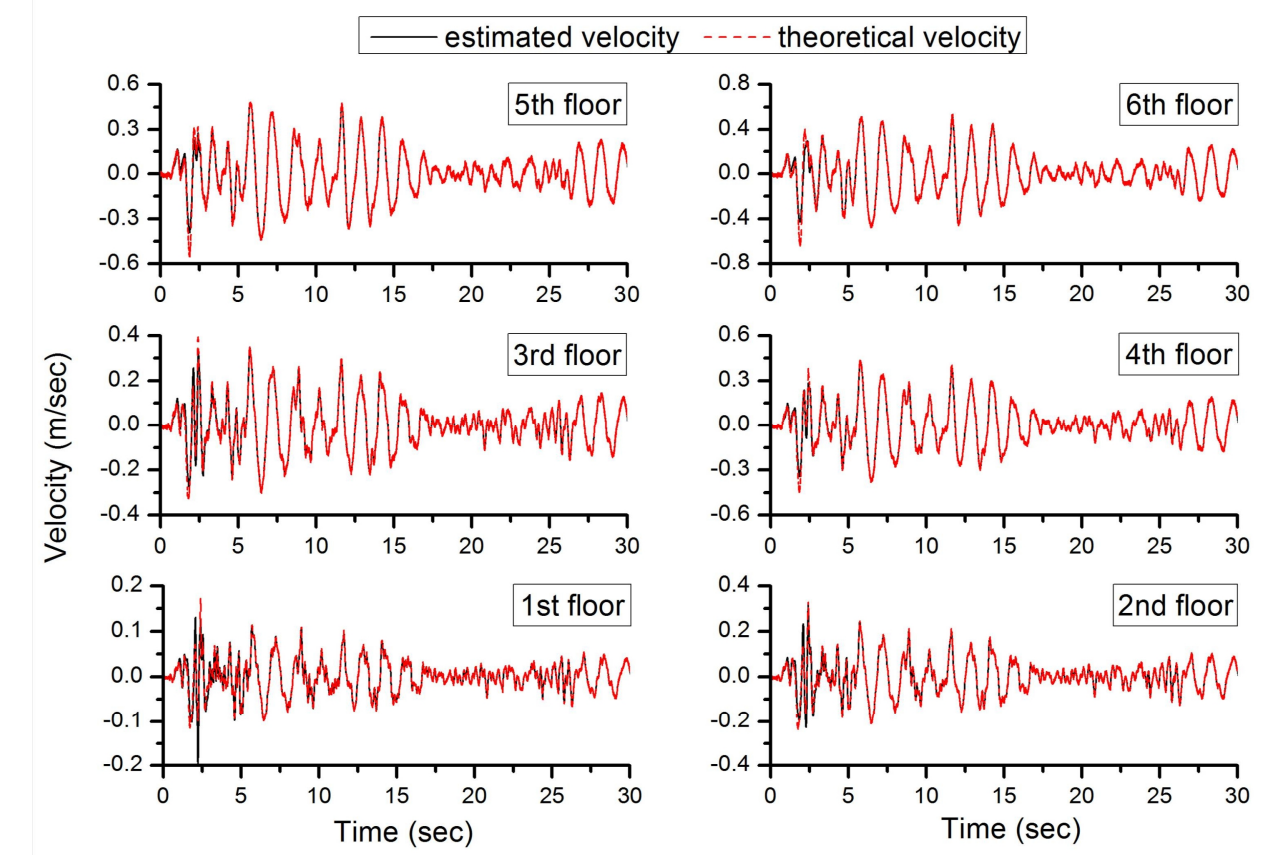

Fig. 4 Time history curves of estimated velocity vs. theoretical velocity with $5 \%$ noise-included case 


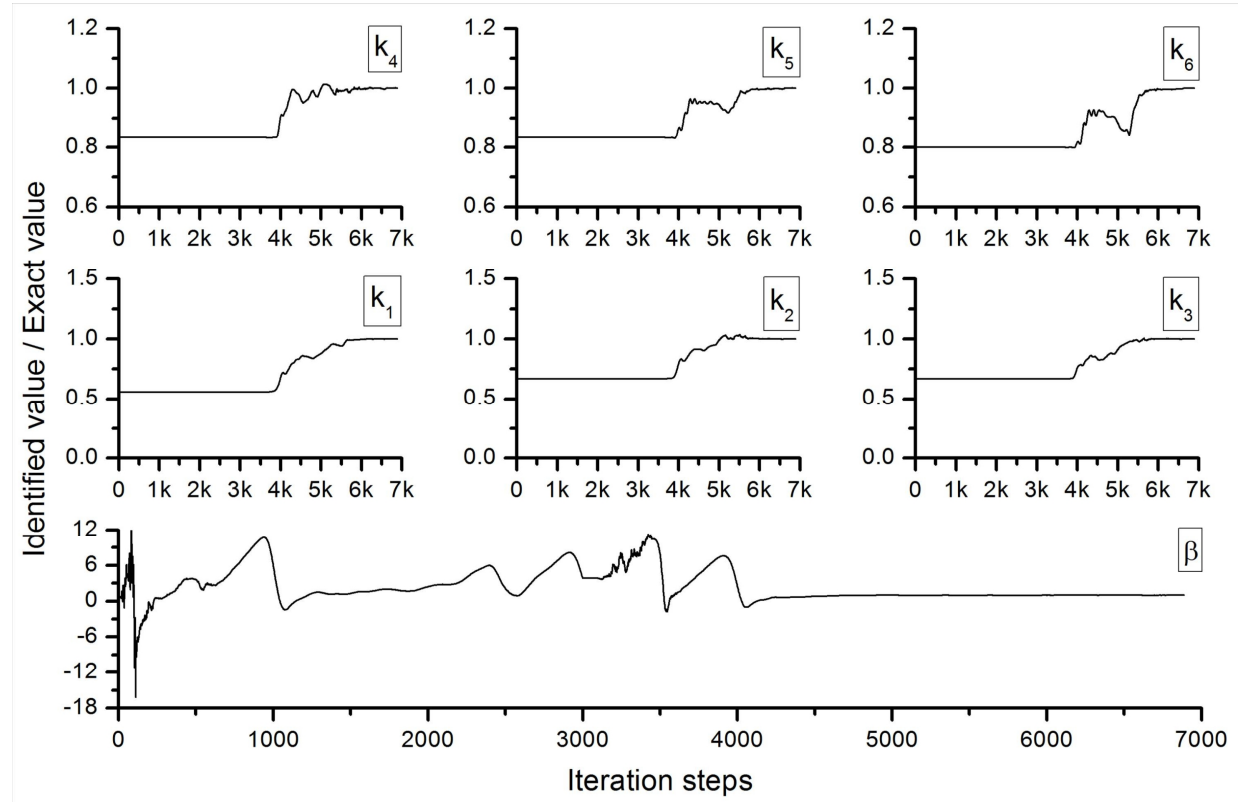

Fig. 5 Convergence process of parameter identification with $5 \%$ noise-included case

In Table 2, the maximum errors of the stiffness parameters and damping coefficient are $0.00 \%$ and $0.00 \%$ in the noise free case, $-0.02 \%$ and $0.22 \%$ in the $1 \%$ noise case, and $-0.17 \%$ and $1.12 \%$ in the $5 \%$ noise case, respectively. Obviously, the proposed MFEKF-WGI technique identifies the structural physical parameters very well.

In Fig. 4, the velocity responses are estimated and compared with the theoretical responses in the $5 \%$ noise contaminated case. The figure show that the estimated responses agree well with the theoretical ones. And the acceleration responses can be given using the motion equations of MDOF system, Eq. 1. So the proposed method estimates the unmeasured responses very well.

In Fig. 5, the convergence processes of parameter identification are given. In the first global iteration, only 3000 sample points are used, the stiffness parameters change little and the damping coefficient varies up and down with the iteration steps. In the second global iteration, the stiffness parameters and damping coefficient reach to the exact values quickly, giving the convergence iteration steps of 6000 in the stiffness parameters and 4000 in the damping coefficient, i.e. the 3000 and 1000 iteration steps of the second global iteration. Although the total number of iteration steps is 18590 for identifying the structural physical parameters and estimating the full unmeasured response time histories, the prosed method requires a small computing time. This is to say, the proposed method estimate the state in a quick convergence speed.

From the results, it is observed that the MFEKF-WGI technique identified the structural physical parameters and estimated the unmeasured responses in time domain very well. There are small errors in the identified parameters and high consistent if the estimated responses with the three noisecontaminated cases. This demonstrates the effectiveness and robustness of the MFEKF-WGI method.

\section{Conclusions}

A structural physical parameter identification technique in time domain, MFEKF-WGI, is proposed to identify structural parameters and estimate the unmeasured responses, where the required output measurements are only the displacement responses at all or most of the DDOFs. The method is verified using a numerical example of 6-story shear-type building. For verification purpose, three noise-included output responses (noise-free, $1 \%$ noise-included case and 5\% noise-included case) are considered.

Several important results are obtained from the numerical example. The proposed MFEKF-WGI procedure identifies the structural physical parameters and estimates the unmeasured responses very well. The errors of the identified parameters and the estimated responses are very small. The proposed method is accurate and robust. 
Since numerical integration is necessary in the KF-WGI procedure, more sample points and smaller time intervals, compared to the ILS (Iterative Lease Squares method), are necessary for the estimated accuracy using the proposed method. In general, the proposed method requires the complete response time histories for estimating the unmeasured responses. The results show that the MFF technique and 4th order R-K method work well and the MFEKF-WGI technique is effective.

\section{Acknowledgements}

The authors are grateful for the financially supported by the National Science Foundation of China (Grant No. 51208478). Any opinions, findings, conclusions and recommendations expressed in this paper are those of the authors and do not necessarily reflect the views of the sponsor.

\section{References}

[1] C.S. Huang, S.L. Hung, C.M. Wen, et al., A neural network approach for structural identification and diagnosis of a building from seismic response data, Earthquake Engineering and Structural Dynamics. 32(2003) 187-206.

[2] Y.F. Shi, C.C. Chang, Substructural time-varying parameter identification using wavelet multiresolution approximation, Journal of Engineering Mechanics. 138(2012) 50-59.

[3] D. Rocco, M. Marco, C.P. Felice, Analysis of non-stationary structural systems by using a band-variable filter, Bulletin of Earthquake Engineering. 10(2012) 895-911.

[4] D. Wang, A. Haldar, Element-level system identification with unknown input, Journal of Engineering Mechanics. 120(1994) 159-176.

[5] X.J. Wang, J. Cui, An iteration algorithm applied for structural physical parameters identification, Earthquake Engineering and Engineering Dynamics. 28(2008) 44-50. (in Chinese)

[6] X.J. Wang, J. Cui, Structural physical parameter identification of nonlinear parametric system in time domain, Applied Mechanics and Materials. $94-96$ (2011) 1040-1043.

[7] X.J. Wang, P. Wan, J. Cui, Structural damage detection and ground motion inversion based on hybrid inversion method, World Earthquake Engineering. 31(2015) 114-121. (in Chinese)

[8] M.S. Gong, J. Sun and L.L. Xie, Identification of model structure parameters via combination of AFMM and ARX from seismic response data, Earthquake Engineering and Engineering Vibration. 13(2014) 411-423.

[9] R. Brincker, L. Zhang, and P. Andersen, Modal identification of output-only systems using frequency domain decomposition, Smart Materials and Structures. 10(2001) 441-445.

[10] D.M. Siringoringo and Y. Fujino, System identification of suspension bridge from ambient vibration response, Engineering Structures. 30(2008) 462-477.

[11] S.R.Ibrahim, Random decrement technique for modal identification of structures, Journal of Spacecraft and Rockets. 14(1977) 696-700.

[12] K. Toki, T. Sato, J. Kiyono, Identification of structural parameters and input ground motion from response time histories, Structural Engineering and Earthquake Engineering. 6(1989) 243-251.

[13] J.Q. Shang, Application of Kalman filter method to dynamic parameter estimation of structures, Earthquake Engineering and Engineering Vibration. 11 (1991) 62-72. (in Chinese)

[14] M. Hoshiya, E. Saito, Structural identification by extended Kalman filter, Journal of Engineering Mechanics, ASCE. 110(1984) 1757-1770.

[15] X. Zhao, J. Li, A weighted global iteration parametric Kalman filter algorithm, Chinese Journal of Computational Mechanics. 19(2002) 403-408. (in Chinese) 
[16] D. Wang, A. Haldar, System identification with limited observation and without input, Journal of Engineering Mechanics, ASCE. 123(1997) 504-511.

[17] X.J. Wang, Study on physical parameter identification of civil engineering in time domain and inversion of ground motion, $\mathrm{PhD}$ Dissertation, Institute of Engineering Mechanics, China Earthquake Administration, 2011. (in Chinese) 